\title{
Des mutations de la superoxyde dismutase à l'origine de formes familiales de sclérose latérale amyotrophique
}

La sclérose latérale amyotrophique est un trouble dégénératif des neurones moteurs du cortex, du tronc cćrébral et de la moelle épinière. Sa cause est inconnue, son évolution inexorable. Environ $10 \%$ des cas sont familiaux (SLAF), reconnaissant une hérédité autosomique dominante. Le tableau clinique des formes familiales et sporadiques est analogue. Siddique et al. (Chicago, IL, USA) avaient montré que dans certaines familles la maladie est liée à un défaut génétique sur le bras long du chromosome 21 [1]. Rosen et al. (Charlestown, MA, USA) ont [2] identifié dans un cosmide une répétition CA appelée D21S223. L'amplification d'un exon (l'exon 2) $\mathrm{du}$ gène de l'enzyme superoxyde dismutase cytoplasmique (SOD1), dont la localisation sur le 21 est connue [3], montra la très grande proximité du gène et du cosmide, ainsi que du locus SLAF. A partir de là, une équipe internationale à dominante nordaméricaine (33 auteurs) a démontré une liaison entre SOD1 et SLAF; le lod score calculé sur des familles de Boston atteignait 6,80 ; la même liaison fut observée dans neuf familles de Chicago [4].

La séquence des cinq exons de la SOD1 est connue [5]. L'analyse de la séquence montra des mutations dans les exons 2 et 4 des malades et aucune chez les 250 témoins. Dans chaque famille comptant un variant, l'haplotype SLAF coségrégeait avec la maladie. En tout, des anomalies furent trouvées dans 18 familles; les exons 2 et 4 furent séquencés dans 13 d'entre elles ; dans tous les cas, on observe une mutation ponctuelle à l'état hétérozygote, permettant de prédire 11 mutations différentes d'acides aminés. Ces mutations sont présentées dans le Tableau I. On peut remarquer qu'une même mutation a été trouvée dans deux familles non apparentées, aux codons 37 et 113 , et que, au niveau de deux codons, 41 et 93 , deux

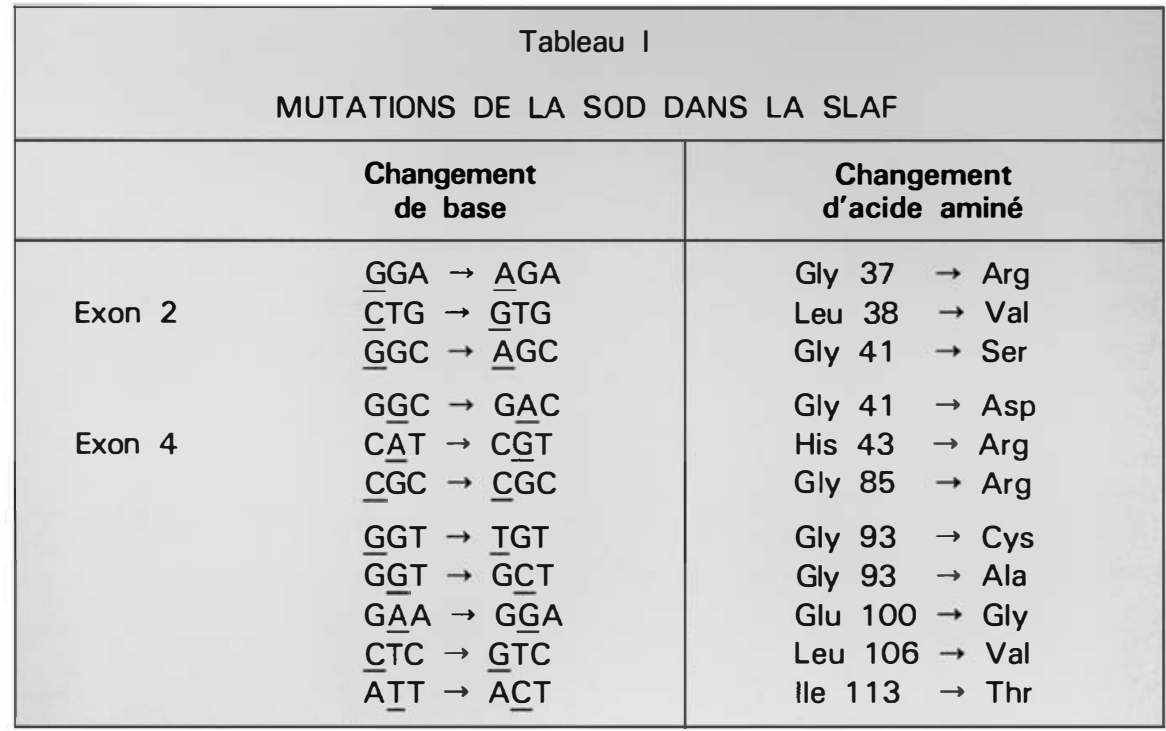

mutations différentes se sont révélées. Pour 9 des 11 mutants, un site de reconnaissance poure une enzyme de restriction était altéré, permettant une identification facile. L'existence de ces nombreuses mutations, chaque fois liées à la présence de la SLAF, si elle ne constitue pas une preuve absolue, rend très probable une relation de cause à effet entre les altérations du gène de la SOD1 et la maladie.

Reste à comprendre comment une altération éventuelle d'une enzyme ubiquitaire comme SOD1 peut conduire aux symptômes précis de la SLA. L'approche de ce problème pourrait être facilitée par les résultats récents de Couratier et al. (Limoges, France) parus dans The Lancet [6]. On savait que le plasma des malades atteints de SLA est toxique pour des neurones en culture. Les auteurs français ont cultivé des neurones d'embryons de rat en présence de liquide céphalorachidien de dix malades et montré sa toxicité. La neurotoxicité était bloquée spécifiquement par un antagoniste du récepteur du kaïnate. Le LCR des malades contient donc un facteur neurotoxique ressemblant à ce récepteur du kaïnate, différent du récepteur NMDA (N- méthylaspartate). En rapprochant les deux types de résultats, on peut parvenir à une hypothèse plausible. La SOD1, qui contient du $\mathrm{Cu}^{2+}$ et du $\mathrm{Zn}^{2+}$, catalyse la conversion du superoxyde en peroxyde d'hydrogène et oxygène : $\mathrm{O}_{2}^{-}+\mathrm{O}_{2}^{-}+2 \mathrm{H}^{+} \rightarrow$ $\mathrm{H}_{2} \mathrm{O}_{2}+\mathrm{O}_{2}$.

$\mathrm{O}_{2}^{-}$apparaît comme un produit d'oxydations spontanées ou catalysées. Il peut agir comme réducteur ou comme oxydant, et le rôle de la SOD est d'en régulariser le flux. La SOD doit être présente là où se forme $\mathrm{O}_{2}$ car ce réactif ne traverse pas les bicouches lipidiques. Le superoxyde réagit aussi avec l'oxyde nitrique NO, dont l'importance s'est révélée récemment $\left(\mathrm{m} / \mathrm{s} n^{\circ} 8\right.$, vol. 8, p. 843), en formant un anion peroxynitrate $\mathrm{ONOO}^{-}$puis un radical toxique $\mathrm{HO}$. Des mutants de bactéries, levure, drosophile, déficients en SOD sont hypersensibles aux dommages oxydatifs. Réciproquement, un excès de SOD réalisé par transgenèse chez la souris conduit à des troubles moteurs de la langue et des membres inférieurs. On peut enfin rappeler qu'il y a une quinzaine d'années on faisait jouer un rôle à la baisse de la SOD dans la sénescence. 
L'hypothèse la plus vraisemblable qui émerge de l'ensemble des données est celle d'un mécanisme excitotoxique entraînant la production de radicaux libres dont l'excès peut provoquer des lésions neuronales. Le principal neuroexcitateur connu est le glutamate ; un lien entre cet acide aminé et une variété de SLA avait été d'ailleurs invoqué dans une forme de démence accompagnant SLA et Parkinson dans l'île de Guam, due à un analogue du glutamate, le BMAA ( $\beta$-N-méthyl aminoalamine) (voir $m / s \quad n^{\circ} 7$, vol. 3, p. 426). Le BMAA exerce ses effets par un récepteur non NMDA. Cette notion, jointe aux résultats de l'équipe française [6], fait que le meilleur candidat récepteur est celui du kaïnate/AMPA*. Le rôle de la SOD serait de protéger les neurones contre un excès de $\mathrm{O}_{\dot{2}^{-}}$, qui pourrait être produit par des enzymes dont le type est la xanthine oxydase, selon un schéma proposé par McNamara et Fridovich [7] (figure 1).

Une altération de la SOD pourrait donc être à l'origine d'une SLA familiale. Cependant, bien des questions se posent encore. La SLAF est une maladie dominante, les 11 mutations différentes trouvées sont à l'état hétérozygote ; il faut supposer que dans tous les cas il y ait une action "dominante négative ", ce qui est possible puisque la forme active de l'enzyme est un dimère. Curieusement, aucune allusion n'est faite au taux de la SOD chez les malades, ni dans l'article de Rosen et al. [4] ni chez les commentateurs [7, 8] ; le dosage est pourtant facile à effectuer sur les globules rouges, et on en a montré par exemple l'augmentation dans la trisomie 21 [3]. Bien entendu, des altérations pourraient être qualitatives, et, dans ce cas, très difficiles à détecter. Autre question, sans doute à l'étude mais non abordée par les auteurs: les cas sporadiques, de loin les plus nombreux, comportent-ils une altération de la SOD? Enfin, dans environ la moitié des familles, le 21 n'est pas en cause. Il reste des candidats SOD, puisqu'on connaît deux autres SOD minoritaires : une SOD à $\mathrm{Mn}^{2+}$ mitochondriale tétramérique dont le gène siège sur le 6 , et une SOD extracellulaire à $\mathrm{Cu}^{2+}$ et $\mathrm{Zn}^{2+}$,

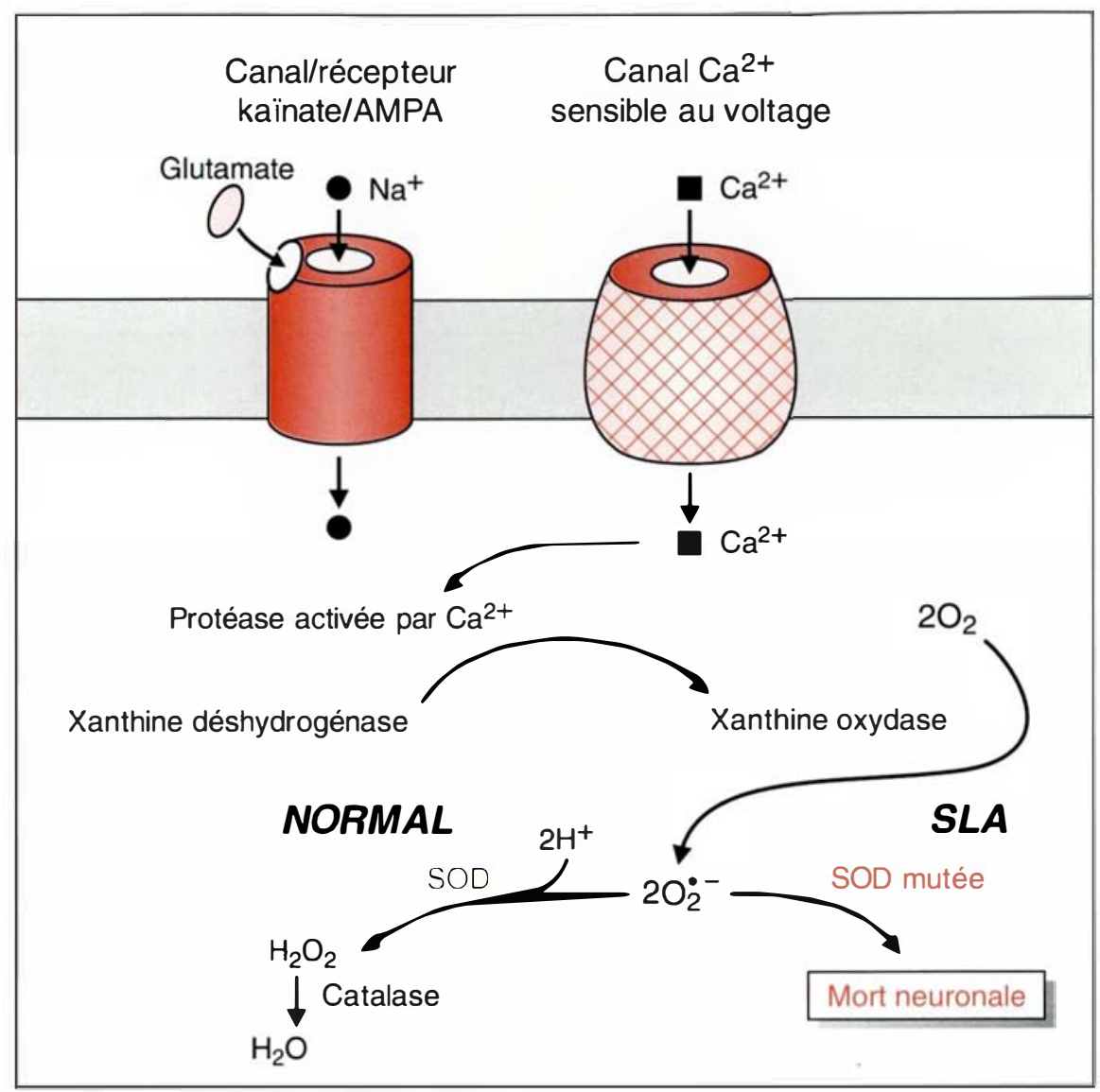

Figure 1. Schéma hypothétique de l'apparition des lésions motoneuronales chez les malades avec forme familiale de SLA liée au chromosome 21. La détoxification anormale de l'anion superoxyde $\mathrm{O}_{\dot{2}}^{-}$chez les malades, conséquence d'une mutation de la superoxyde dismutase (SOD) cytosolique, entraîne la mort des motoneurones. (Modifiée d'après [7].)

tétramérique, dont le siège du gène n'est pas connu. Beaucoup d'efforts donc encore en perspective, d'autant que les anomalies des SOD n'ont sans doute pas le monopole dans la genèse de la SLA. Cependant, le pas franchi par la découverte de Rosen et al. est considérable, tant au point de vue théorique que pratique; on peut en effet envisager des voies thérapeutiques : par exemple, utiliser des antagonistes des récepteurs du kainate, ou des agents susceptibles d'éliminer $\mathrm{O}_{2}^{-}$, comme les vitamines $\mathrm{C}$ ou $\mathrm{E}$, ou des produits plus efficaces capables de les remplacer.

J.C.D.

1. Siddique T, Figlewicz DA, PericakVance MA, et al. Linkage of a gene causing familial amyotrophic lateral sclerosis to chromo- some 21 and evidence of genetic-locus heterogeneity. $N$ Engl J Med 1991; 324: 1381-4.

2. Rosen DR, Sapp PC, O'Regan J, et al. Dinucleotide repeat polymorphisms (D21S223 and D21S224) at 21q22.1. Hum Mol Genet $1992 ; 1$ : 547

3. Sinet PM, Nicole A, Ceballos I, Delabar JM. Trisomie 21 et maladie d'Alzheimer. médecinessiences $1987 ; 3: 256-62$.

4. Rosen DR, Siddique T, Patterson D, et al. Mutations in $\mathrm{Cu} / \mathrm{Zn}$ superoxide dismutase gene are associated with familial amyotrophic slerosis. Nature 1993 ; 362 : 59-62.

5. Levanon D, Lieman-Hurwitz J, Dafni N, et al. Architecture and anatomy of the chromosomal locus in human chromosome 21 encoding the $\mathrm{Cu} / \mathrm{Zn}$ superoxide dismutase. $E M B O J 1985$; 4: 66-84.

6. Couratier P, Hugon J, Sindou P, Vallat JM, Dumas M. Cell culture evidence for neuronal degeneration in amyotrophic lateral sclerosis being linked to glutamate AMPA/kainate receptors. Lancet 1993 ; 341 : 265-8

7. McNamara J, Fridovich I. Did radicals strike Lou Gehrig. Nature 1993 ; 362 : 20-1.

8. Marx J. Gene linked to Lou Gehrig disease. Science $1993 ; 259 ; 1393$. 